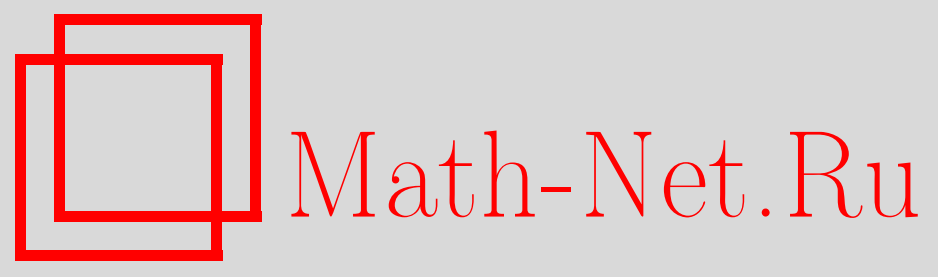

В. И. Сенашов, Строение бесконечной силовской подгруппы в некоторых периодических группах Шункова, Дискрет. матем., 2002, том 14, выпуск 4, 133-152

DOI: https://doi.org/10.4213/dm268

Использование Общероссийского математического портала Math-Net.Ru подразумевает, что вы прочитали и согласны с пользовательским соглашением http://www.mathnet.ru/rus/agreement

Параметры загрузки:

IP : 54.89 .56 .158

26 апреля 2023 г., 13:34:19

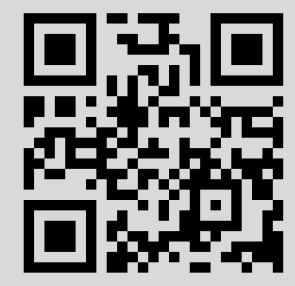




\title{
Строение бесконечной силовской подгруппы в некоторых периодических группах Шункова
}

\author{
() 2002 г. В. И. Сенашов
}

\begin{abstract}
Изучаются периодические группы, для которых нормализатор любой конечной нетривиальной подгруппы почти слойно конечен. Класс групп, удовлетворяющих этому условию довольно широк, в нем содержатся свободные бернсайдовские группы нечетного периода, большего 665, и группы, построенные А. Ю. Ольшанским.

Рассматривается классический вопрос: как свойства системы подгрупп влияют на свойства всей группы? Показывается, что почти слойная конечность распространяется на группу $G$ с нормализаторов нетривиальных конечных подгрупп группы $G$, когда $G$ является периодической сопряженно бипримитивно конечной группой, обладающей сильно вложенной подгруппой. Изучается строение бесконечной силовской 2-подгруппы в периодической сопряженно бипримитивно конечной группе, когда в группе нормализатор любой конечной нетривиальной подгруппы почти слойно конечен.

Результаты статьи могут также быть полезными при изучении класса периодических сопряженно бипримитивно конечных групп (периодических групп Шункова).

Работа выполнена при поддержке Российского фонда фундаментальных исследований, проекты 99-01-00432, 02-01-00078, и гранта №9 Шестого конкурса научных проектов молодых ученых 1999 г.
\end{abstract}

\section{1. Введение}

Слойно конечные группы впервые появились без названия в статье С. Н. Черникова [1], а затем в его последующих публикациях за ними закрепилось название слойно конечных групп. Группа называется слойно конечной, если множество ее элементов любого данного порядка конечно. Почти слойно конечные группы - это конечные расширения слойно конечных групा.

В этой статье мы изучаем периодические группы, для которых нормализатор любой конечной нетривиальной подгруппы почти слойно конечен. Класс групп, удовлетворяющий этому условию, довольно широк, в нем содержатся свободные бернсайдовские групшы нечетного периода, большего 665 (см. [2]), и группы, построенные А. Ю. Ольшанским (см. [3]).

В теореме 1 рассматривается классический вопрос: как свойства системы подгрупп влияют на свойства всей группы? Показывается, что почти слойная конечность распространяется на групту $G$ с нормализаторов нетривиальных конечных подгрупп группы $G$, 
когда $G$ является периодической сопряженно бипримитивно конечной группой, обладающей сильно вложенной подгруппой.

Изучается строение бесконечной силовской 2-подгруппы в периодической сопряженно бипримитивно конечной группе (теорема 2), когда в группе нормализатор любой конечной нетривиальной подгрупшы почти слойно конечен.

Результаты статьи могут также быть полезными при изучении класса периодических сопряженно бипримитивно конечных групп. За такими группами закрепилось название периодических групп Шункова. Этот термин уже используется в работах В. И. Сенашова, А. И. Созутова, А. К. Шлепкина, А. В. Рожккова, Л. Хаммуди. В частности, Л. Хаммуди построен новый пример простой бесконечной конечно порожденной сопряженно бипримитивно конечной $p$-групты, что подчеркивает важность результатов настоящей статьи.

Ранее автором была установлена почти слойная конечность периодической группы Шункова без инволюций с указанным выше условием $[4,5]$.

Целью настоящей работы является доказательство следующих теорем.

Теорема 1. Пусть периодическая группа Шункова $G$ содержит сильно вложенную подгруппу. Если в $G$ нормализатор любой нетривиальной конечной подгруппы почти слойно конечен, то и сама группа $G$ почти слойно конечна.

Теорема 2. Пусть в периодчческой не почти слойно конечной группе Шункова нормализатор любой нетривиальной конечной подгруппы почти слойно конечен. Если силовская 2-подгруппа группы $G$ бесконечна, то она является квазидиэдральной 2-группой.

Квазидиэдральной 2-группой называется расширение квазициклической 2-группы при помощи обращающего автоморфизма (название объясняется тем, что эта группа является объединением 2-групп диэдра).

\section{2. Некоторые определения}

Напомним определения специальных терминов, использующихся в работе.

Группа $G$ называется группой Шункова (сопряженно бипримитивно конечной группой), если для любой ее конечной подгруппы $H$ в фактор-группе $N_{G}(H) / H$ любые два сопряженных элемента простого порядка порождают конечную подгрупту.

Группа называется слойно конечной, если множество ее элементов любого данного порядка конечно.

Подтруппа $H$ группы $G$ называется бесконечно изолированной в $G$, если $H$ содержит централизаторы всех своих элементов простых порядков, имеющих бесконечные пересечения с $H$.

Подгруппа $H$ группы $G$ называется сильно вложенной в $G$, если $H$ - собственная подгруппа группы $G$, содержащая инволюции, и $H \cap x^{-1} H x$ не содержит инволюций для $x \in G \backslash H$.

Элемент с конечным централизатором в группе $G$ называется почти регулярным элементом группы $G$.

Элемент второго порядка называется инволюцией. 


\section{3. Доказательство основных результатов}

Пусть периодическая группа Шункова $G$ не является почти слойно конечной, а нормализатор любой нетривиальной конечной подгруппы группы $G$ почти слойно конечен.

Предварим доказательства теорем рядом лемм.

Лемма 1. Группа $G$ не является примарной группой и силовские $p$-подгруппы в $G$ черниковские.

Доказательство. Пусть $P-p$-подгруппа группы $G$. Среди ее элементарных абелевых подгрупп, очевидно, найдется максимальная подгруппа $R$. Бесконечной группа $R$ быть не может ввиду условий, накладываемых на группу $G$. Следовательно, любая абелева подгруппа из $P$ имеет конечный нижний слой. Тогда, как известно (см., например, [7]), абелевы подгрупшы удовлетворяют условию минимальности и по основному результату из [12] $P$ - черниковская группа. Если же $G$ является $p$-группой, то по только что доказанному она должна быть черниковской группой, что невозможно ввиду выбора контрпримера. Получаем противоречие. Лемма доказана.

Лемма 2. Не нарушая общности рассуждений, можно предполагать, что группа $G$ не обладает неединичным локально конечным радикалом.

Доказательство. Если локально конечный радикал $L(G) \neq 1$, то он почти слойно конечен ввиду условий, налагаемых на группу $G$, и ввиду почти слойной конечности локально конечной подгруппы, удовлетворяющей условиям, налагаемым на группу $G$ (см. [20]). Следовательно, в нем найдется конечная характеристическая подгруппа, нормализатор которой в силу условий, налагаемых на группу $G$, почти слойно конечен. Получили противоречие с выбором контрпримера. Лемма доказана.

В дальнейшем считаем в соответствии с леммой 2 , что в $G$ локально конечный радикал тривиален.

Лемма 3. Любая локально конечная подгруппа из $G$ вкладывается в максимальную почти слойно конечную подгруппу из $G$.

Доказательство. Среди всех локально конечных подгрупп группы $G$, содержащих данную подгруппу, по лемме Цорна найдется максимальная. Ввиду теоремы Шункова из [20] она будет почти слойно конечной. Лемма доказана.

Лемма 4. Пусть $F, M$ - две различные бесконечные максимальные почти слойно конечные подгруппы группы $G, R(F)$ u $R(M)$ - их слойно конечные радикалы.

Тогда

$$
R(F) \cap R(M)=1
$$


Доказательство. Пусть

$$
b \in R(F) \cap R(M), \quad b \neq 1 .
$$

Если $R(F) \cap R(M)$ имеет конечные индексы в $F$ и $M$, то по предложению 8 из [8] и почти слойной конечности локально конечной группы, удовлетворяющей условиям теоремы 1 (см. [20]), получаем противоречие с максимальностью $F$ (по лемме Дищмана в почти слойно конечной группе всегда найдется конечная характеристическая подгрупта).

Пусть тогда для одной из подгрупп, например, для $M$

$$
|M: R(M) \cap R(F)|=\infty .
$$

Подгруппа $C=C_{G}(b)$ ввиду свойств слойно конечных групп пересекается с $F$ и $M$ по подгруппам конечного индекса. Подгруппа $C$ почти слойно конечна в силу условий, налагаемых на группу $G$. Слойно конечный радикал $R(C)$ группы $C$, очевидно, также пересекается с $F$ и $M$ по подгруппам конечного индекса. Так как

$$
|M: R(C) \cap M|<\infty,
$$

a

$$
|M: R(F) \cap M|=\infty,
$$

радикал $R(C)$ не лежит в $F$. Ввиду слойной конечности группы $R(C)$, в ней найдется подгруппа $B$ такая, что

$$
F \cap R(C)=F \cap B, \quad|B: F \cap B|<\infty .
$$

По предложению 8 из [8] существует подгруппа $T \leqslant B \cap F$, в нормализатор которой входят подгруппы $F$ и $B$. Из максимальности подгруппы $F$ получаем, что $G=N_{G}(T)$. Но группа $G$ неединичным локально конечным радикалом не обладает по лемме 2 . Получаем противоречие. Лемма доказана.

Лемма 5. Любая максимальная почти слойно конечная подгруппа из $G$ является бесконечно изолированной подгруппой.

Доказательство. Обозначим через $H$ произвольную максимальную почти слойно конечную подгруппу группы $G$. Предположим, что утверждение леммы неверно и найдется элемент $a \in H$ простого порядка, для которого $C_{G}(a)$ не лежит в $H$ и в то же время

$$
\left|C_{G}(a) \cap H\right|=\infty \text {. }
$$

Очевидно, пересечение $C_{G}(a) \cap R(H)=D$ бесконечно. Включим $C_{G}(a)$ в максимальную почти слойно конечную подгруппу $B$. Тогда $D \subset H \cap B$. Так как

$$
|B: R(B)|<\infty \text {, }
$$

то и индекс

$$
|D: R(B) \cap D|
$$

конечен (напомним еще, что $D<R(H)$ ). Следовательно, пересечение $R(H) \cap R(B)$ нетривиально и $B=H$ по лемме 4. Получаем противоречие. Лемма доказана. 
Лемма 6. Пусть $b$ - элемент простого порядка и пересечения

$$
C_{G}(b) \cap H, \quad C_{G}(b) \cap H^{g}
$$

бесконечны, где $H$ - максимальная почти слойно конечная подгруппа группы $G$.

Тогда

$$
H=H^{g} \text {. }
$$

Доказательство. По лемме 5

$$
C_{G}(b) \subset H, \quad C_{G}(b) \subset H^{g} .
$$

Следовательно,

$$
C_{G}(b) \subset H \cap H^{g} .
$$

Так как $C_{G}(b)$ бесконечен, то $R(H) \cap C_{G}(b)$ - бесконечная группа и

$$
\left|C_{G}(b): R(H) \cap C_{G}(b)\right|<\infty \text {. }
$$

Точно так же

$$
\left|C_{G}(b): R\left(H^{g}\right) \cap C_{G}(b)\right|<\infty .
$$

Тогда пересечение $R(H) \cap R\left(H^{g}\right)$ обладает неединичным элементом. Используя лемму 4, получаем доказываемое утверждение.

Лемма 7. Все инволючии в $G$ имеют бесконечные чентрализаторы.

Доказательство. Предположим, что некоторая инволюция обладает конечным централизатором в группе $G$. Тогда по известной теореме Шункова из [6] группа $G$ локально конечна, что противоречит почти слойной конечности локально конечной подгруппы ввиду условий, налагаемых на группу $G$ (см. [20]). Лемма доказана.

Лемма 8. В максимальной почти слойно конечной подгруппе $V$ из $G$ все инволючии $c$ бесконечными чентрализаторами в $V$ порождают конечную подгруппу.

Доказательство. Предположим, что это не так и группа, порожденная инволюциями из $V$ с бесконечными централизаторами в $V$, бесконечна. Ввиду строения почти слойно конечной группы и леммы Дицмана в этом случае в $V$ найдется инволюция $i$ с бесконечным $C_{V}(i)$, для которой индекс $\left|V: C_{V}(i)\right|$ бесконечен. Обозначим через $\mathfrak{\Re}$ класс инволюций из $V$, сопряженных с $i$ в $V$. Для произвольного элемента $g \in G \backslash V$ рассмотрим подгруппу

$$
V^{g}=g^{-1} V g
$$

и ее подмножество

$$
\mathfrak{M}=\mathfrak{l}^{g}=g^{-1} \mathfrak{N} g .
$$

Ввиду того, что $G$ является группой Шункова, любые две инволюции из множеств $\mathfrak{⿰}$ и $\mathfrak{M}$ порождают конечные подгруппы. Тогда для произвольной фиксированной инволюции $x$ из $\mathfrak{N}$ элементы $b_{t}=x t, t \in \mathfrak{M}$, имеют конечные порядки. 
Если для бесконечного подмножества $\mathfrak{U}$ из $\mathfrak{M}$ порядки элементов $b_{t}, t \in \mathfrak{U}$, нечетны, то по свойствам групп диэдра в $\left(b_{t}\right)$ найдется элемент $c_{t}$ такой, что $c_{t}^{-1} t c_{t}=x$. Так как

$$
t \in \mathfrak{U} \leqslant \mathfrak{M}
$$

то $t=g^{-1} r g$ для некоторой инволюции $r$ из $\mathfrak{\Re}$. Отсюда получим, что

$$
c_{t}^{-1} g^{-1} r g c_{t}=x .
$$

Обозначая $h_{t}=g c_{t}$, видим, что

$$
x \in h_{t}^{-1} V h_{t}=V_{t}
$$

По определению множества $\mathfrak{U}$ инволюции $x$ и $r$ сопряжены с $i$ в $V$ и по предположению имеют бесконечные централизаторы в $V$. Отсюда централизатор инволюции $x$ в $V$ также бесконечен и по лемме 5

$$
C_{G}(x)<V \cap V_{t}
$$

Тогда $V=V_{t}$ по лемме 6. Ввиду максимальности $V$ и по свойствам группы $G$

$$
h_{t} \in V=N_{G}(V) \text {. }
$$

Элемент $g$ можно представить в виде

$$
g=h_{t} c_{t}^{-1}, \quad t \in \mathfrak{U},
$$

тогда

$$
V g=V c_{t}^{-1}, \quad t \in \mathfrak{U} .
$$

Для двух различных инволюций $t_{1}, t_{2}$ из $\mathfrak{U}$ соответствующие строго вещественные элементы $c_{t_{1}}, c_{t_{2}}$ также различны. Иначе из их совпадения вытекало бы равенство

$$
c_{t_{1}}^{-1} t_{1} c_{t_{1}}=c_{t_{2}}^{-1} t_{2} c_{t_{2}}
$$

что невозможно для различных $t_{1}, t_{2}$. По свойствам групп диэдра элемент $j_{t}=x c_{t}^{-1}$ из $V g$ есть инволюция. Множество таких инволюций по мощности совпадает с мощностью множества $\mathfrak{U}$ и, значит, бесконечно. В качестве представителя смежного класса $V g$ берем инволюцию $k=x c_{t}^{-1}$ для некоторого $t$ из $\mathfrak{U}$. Тогда инволюцию $j_{t}$ можно представить в виде

$$
j_{t}=s_{t} k, \quad t \in \mathfrak{U},
$$

где $s_{t} \in V$ и является строго вещественным относительно инволюции $k$ ввиду равенства

$$
\left(s_{t} k\right)^{2}=\left(j_{t}\right)^{2}=1
$$

отсюда

$$
k^{-1} s_{t} k=s_{t}^{-1} \text {. }
$$

Очевидно, что группа

$$
Z=\operatorname{gr}\left(s_{t} \mid t \in \mathfrak{U}\right)
$$


бесконечна и $Z<V$. Инволюция $k$ нормализует $Z$ и не лежит в $V$. Включим почти слойно конечную подгруппу $N_{G}(Z)$ в максимальную почти слойно конечную подгруппу $M$ группы $G$ (это можно сделать по лемме Цорна ввиду почти слойной конечности локально конечных подгрупп в силу условий, налагаемых на группу $G$ (см. [20])). Пересечение $V \cap M$ бесконечно (в нем содержится подгруппа $Z$ ). Отсюда по лемме 4 получаем совпадение $V=M$ и включение $k \in V$ вопреки выбору $k$.

Противоречие означает, что для любого элемента $x \in \mathfrak{N}$ найдется бесконечное подмножество $\mathfrak{U}_{x}$ множества $\mathfrak{M}$ такое, что порядки элементов

$$
b_{t}=x t, \quad t \in \mathfrak{U}_{x},
$$

четны. Обозначим через $\mathfrak{B}$ множество инволюций вида

$$
j_{t} \in\left(b_{t}\right), \quad t \in \mathfrak{H}_{x} .
$$

По свойствам групп диэдра и лемме 5

$$
\mathfrak{B} \leqslant V \cap V^{g}
$$

Ввиду максимальности $V$ из бесконечности множества $\mathfrak{B}$ следовало бы по лемме 4 совпадение $V=V^{g}$, что противоречило бы выбору пары $V, g$. Следовательно, $\mathfrak{B}$ - конечное множество и, не нарушая общности рассуждений, будем считать, что оно состоит из одной инволюции $j_{x}$. По свойствам групп диэдра $(x)$

$$
\mathfrak{U}_{x} \subset C_{G}\left(j_{x}\right)
$$

и $\mathfrak{U}_{x}-$ бесконечное множество инволюций из $V^{g}$. По лемме 5

$$
x \in C_{G}\left(j_{x}\right) \leqslant V^{g} .
$$

Отсюда ввиду произвольности выбора инволюции $x$ из $\mathfrak{N}$ получаем, что

$$
\mathfrak{i} \leqslant V \cap V^{g} .
$$

Как и выше, в такой ситуации приходим к противоречию с выбором пары $V, g$. Лемма доказана.

Лемма 9. В максимальной почти слойно конечной подгруппе $V$ из $G$ нет элементарной абелевой подгруппы восьмого порядка с почти регулярной инволючией в $V$.

Доказательство. Пусть лемма неверна и $F$ - подгруппа восьмого порядка из $V, j$ - ее почти регулярная в $V$ инволюция.

Так как в бесконечной локально конечной группе четверная подгруппа Клейна обладает инволюцией с бесконечным централизатором (см., например, [9]), в $F$ найдется инволюция с бесконечным централизатором в $V$. Пусть это будет $i$. Аналогично, так как $F=(i) \times K$, где $K$ - группа диэдра, можно считать, что некоторая инволюция $l$ также не является почти регулярной в $V$. Так как $i$ находится по лемме 8 в конечном нормальном в $V$ делителе $V_{i}$, а $l$ соответственно в конечном нормальном в $V$ делителе $V_{l}$, их 
произведение $i l$ также попадает в конечный нормальный делитель $V_{i} V_{l}$ и $i l$ также имеет бесконечный централизатор в $V$. Таким образом, подгруппа

$$
L=(i) \times(l)
$$

имеет бесконечный централизатор в $V$. Теперь рассмотрим максимальную почти слойно конечную в $G$ подгрупту $M$, содержащую $C_{G}(j)$. Очевидно, что

$$
F<C_{G}(j) \leqslant M \text {. }
$$

Как и выше, найдем в $F$ подгруппу $L_{1}$ четвертого порядка с бесконечным централизатором в $M$. Рассматривая тройку подгрупп $L, L_{1}, F$, легко заметить, что пересечение $L \cap L_{1}$ содержит некоторую инволюцию, централизатор которой лежит в $V \cap M$. Так как централизатор любой инволюции в $G$ бесконечен, подгруппы $V, M$ пересекаются по бесконечной подгруппе, и значит, по своим слойно конечным радикалам. Получаем противоречие с леммой 4. Лемма доказана.

Лемма 10. В почти слойно конечной группе $V$ существует лишь конечное число несопряженных конечных разрешимых подгрупп заданного порядка.

Доказательство. Для черниковской группы утверждение леммы следует из теоремы Черникова (см., например, [19]). Пусть теперь $V$ - нечерниковская группа.

Пусть сначала в группе $V$ найдется бесконечное множество элементарных абелевых $q$-подгрупп порядка $k$

$$
L_{1}, L_{2}, \ldots, L_{n}, \ldots
$$

Включим группу $L_{n}$ в силовскую $q$-подгруппу $Q_{n}$ из $V, n=1,2, \ldots$ По лемме 1 силовские примарные подгруппы в $V$ черниковские, поэтому к $V$ можно применить теорему из [13], по которой все силовские $q$-подгруппы $Q_{1}, Q_{2}, \ldots$ сопряжены в локально конечной группе $V$ с черниковскими примарными подгруппами. Так как в локально конечной группе с черниковскими примарными подгруппами силовские $q$-подгруппы сопряжены, внутри $Q_{n}, n=1,2, \ldots$, найдется лишь конечное число несопряженных подгрупп порядка $k$ и для элементарных абелевых подгрупп утверждение леммы доказано. Пусть теперь

$$
L_{1}, L_{2}, \ldots, L_{n}, \ldots
$$

- последовательность разрешимых подгрупп заданного порядка $k$. Доказательство проведем индукцией по $k$. Так как все подгруппы последовательности разрешимы, они обладают нормальными элементарными абелевыми подгруппами

$$
Q=Q_{1}, Q_{2}, \ldots, Q_{n}, \ldots
$$

соответственно. По доказанному выше среди них лишь конечное число несопряженных в $V$. Не нарушая общности рассуждений, будем считать, что все они сопряжены с $Q$, то есть

$$
Q_{n}^{c_{n}}=Q, \quad c_{n} \in V, \quad n=1,2, \ldots
$$

Рассмотрим $A=N_{V}(Q)$. Очевидно, что

$$
L_{n}^{c_{n}} \leqslant A, \quad\left|L_{n}^{c_{n}} / Q\right| \leqslant k .
$$


По свойствам почти слойно конечных групп $A / Q$ - почти слойно конечная группа и по индуктивному предположению среди

$$
L_{1}^{c_{1}} / Q, L_{2}^{c_{2}} / Q, \ldots, L_{n}^{c_{n}} / Q, \ldots
$$

имеется только конечное число несопряженных в $A / Q$ подгрупп. Но тогда такое же утверждение верно и для подгрупп начальной последовательности. Лемма доказана.

Лемма 11. Множество несопряженных элементарных абелевых подгрупп из почти слойно конечной группы $V$ с конечными чентрализаторами в $V$ конечно.

Доказательство. Ввиду того, что в слойно конечной группе централизатор любого элемента имеет конечный индекс, нам достаточно рассмотреть только элементарные абелевы $q$-подгруппы для

$$
q \in \pi=\pi(V \backslash R(V)) .
$$

Поскольку $\pi$ - конечное множество, а порядки элементарных абелевых $q$-подгрупп из $V$ не могут расти неограниченно для каждого $q$ из $\pi$, имеется только конечное число вариантов для порядков таких подгрупп. Отсюда по лемме 8 получаем утверждение леммы.

Лемма 12. Пусть $V$-максимальная почти слойно конечная подгруппа в $G$, содержащая инволючии. Тогда

(1) все инволючии с бесконечными чентрализаторами в $V$ сопряжены в $V$;

(2) если $k$ - инволючия из $V$ и $C_{V}(k)$ конечен, то $k$ индуцирует автоморфизм в некоторой абелевой нормальной подгруппе конечного индекса из $V$, переводящий каждый элемент этой подгруппы в обратный.

Доказательство. Докажем утверждение (1). Пусть $i, k$ - некоторые инволюции из $R(V)$, не сопряженные в $V$ и имеющие в ней бесконечные централизаторы. Рассмотрим группу

$$
D=\operatorname{gr}(i, t)
$$

где $t=k^{g} \notin V$. В случае нечетности порядка элемента $i t$ группа $D$ была бы группой Фробениуса и вопреки предположению $i, t$ были бы сопряжены. Значит, $i t$ - элемент четного порядка. Обозначим через $j$ инволюцию из (it). По свойствам групп диэдра $j$ является центральной инволюцией в $D$ и, следовательно, лежит в $V$ ввиду ее бесконечной изолированности (лемма 5). Обозначим через $S$ силовскую 2-подгруппу из $V$, содержащую $i$ и $j$. Так как в $V$ все силовские 2-подгруппы сопряжены, можно считать, не нарушая общности рассуждений, что $k$ также лежит в $S$, причем $i \neq k$, иначе получили бы противоречие с предположением.

Инволюция $j$ имеет конечный централизатор в $V$, так как иначе ввиду бесконечной изолированности $V$ инволюция $t$ попала бы в $V$ вместе с $C_{G}(j)$.

По лемме 7 элементарная абелева 2-подгруппа, содержащая $j$, не может иметь порядок, больший четырех. Рассмотрим максимальную элементарную абелеву подгруппу $R=(i) \times(j)$ из $S$. Предположим, что все инволюции из $S$ порождают абелеву группу. 
Тогда $k \in R$, иначе существовала бы элементарная абелева группа восьмого порядка в $V$. Отсюда по лемме 8 ввиду строения почти слойно конечной группы следует, что $j$ должна иметь конечный централизатор в $V$. Получаем противоречие с почти регулярностью $j$ в $V$.

Следовательно, инволюции из $S$ порождают неабелеву группу. Если инволюция $i$ не лежит в $Z(S)$, то ввиду максимальности $R$ центральная инволюция из $S$ совпадает либо с $j$, либо с $i j$. В первом случае $j$ попадает в слойно конечный радикал группы $V$, а во втором случае, как и выше, получаем противоречие с почти регулярностью $j$ в $V$. Таким образом, $i \in Z(S)$.

Рассмотрим максимальную в $G$ почти слойно конечную подгруппу $M$, содержащую $C_{G}(t)$. Пусть теперь $D_{1}=\operatorname{gr}\left(i^{g_{1}}, t\right)$ взята так, что $i^{g_{1}} \notin M$. Рассмотрим силовскую 2-подгруппу $P$ из $M$, содержащую инволюцию $t$ и центральную инволюцию $j_{1}$ из $D_{1}$ $\left(D_{1}\right.$, как и выше, не является группой Фробениуса). Инволюция $j_{1}$ попадет вместе с $C_{G}(t)$ в $M$. Мы имеем ситуацию, совершенно симметричную началу доказательства леммы с гругтой $D$. Как и в том случае, $j_{1}$ почти регулярна в $M$ и по лемме $7 R_{1}=(t)\left(j_{1}\right)$ является максимальной элементарной абелевой подгруппой в $P$. Это сразу влечет принадлежность центральной инволюции из $P_{1}$ подгруппе $R_{1}$. Как и ранее, сразу же исключаются возможности инволюциям $j_{1}$ и $t j_{1}$ быть центральными в $P$ ввиду их почти регулярности в $M$. Таким образом, $j \in Z(P)$. Теперь заметим, что $M=V^{g}$. Действительно, $V^{g}$ содержит в слойно конечном радикале элемент $t=k^{g}$. Его же содержит в своем слойно конечном радикале и $M$. По лемме 4 получаем, что $M=V^{g}$. Теперь, учитывая сопряженность силовских подгрупп в $M$, получаем сопряженность между собой нижних слоев центров силовских подгрупп $S$ и $P$. Снова используя лемму 7, видим, что они совпадают с $(i)$ и (j) соответственно. Тем самым утверждение (1) доказано.

Докажем утверждение (2). Предположим, что $C_{V}(k)$ конечен. В этом случае $k \notin R(V)$. Если силовские $p$-подгруппы из $R(V)$ конечны, то рассмотрим пересечение

$$
R(V) \cap C_{G}(k) .
$$

Это пересечение конечно и для любого элемента из него по теореме Каргаполова из [10] найдется нормальный делитель конечного индекса в $R(V)$, не содержащий этого элемента. Тогда пересечение всех таких нормальных делителей по теореме Пуанкаре само будет иметь конечный индекс в $V$ (таких нормальных делителей конечное число). Если теперь взять его пересечение с $R(V)$, то получим нормальную подгруппу $U$ конечного индекса в $V$. Инволющия $k$ действует на $U$ регулярно и, значит, по предложению 4.2 из [19] строго вещественно. Таким образом, для конечных силовских подгрупп утверждение (2) доказано.

Пусть теперь в $V$ имеются бесконечные силовские подгруппы. Тогда ввиду строения почти слойно конечных групп $V$ обладает нетривиальной полной частью $\tilde{V}$. Рассмотрим группу $\tilde{V} \lambda(k)$. Так как $k$ имеет конечный централизатор в $V$, по предложению 7 из [6] и ввиду строения полной части группы $V$ инволюция $k$ переводит любой элемент из $\tilde{V}$ сопряжением в обратный. В фактор-группе $V / \tilde{V}$ все силовские подгруппы конечны. Как и выше для случая конечных силовских подгрупп, найдем нормальную подгруппу $Q$ конечного индекса в $V / \tilde{V}$, состоящую из строго вещественных элементов относительно $k \tilde{V}$. По предложению 4.2 из [19] $Q$ - абелева группа. Очевидно, выбросив из $Q$ силовские 
подгруппы по простым числам из $\pi(Q) \cap \pi(\tilde{V})$, опять получаем абелеву нормальную подгруппу конечного индекса в $V / \tilde{V}$. По предложению 4.2 из [19] и ввиду того, что $V$ - локально конечная $(\pi(Q))^{\prime}$-группа, ее полный прообраз в $V$ также является абелевой нормальной в $V$ подгруппой конечного индекса, состоящей из строго вещественных относительно $k$ элементов. Лемма доказана.

Пусть $S$ - некоторая бесконечная силовская 2-подгруппа из $G, i$ - центральная инволюция из $S$ (группа $S$ является черниковской по лемме 1), причем в качестве $i$ выбираем центральную инволюцию $i$ из полной части группы $S$ (по свойствам черниковских примарных групп, в них пересечение центра и полной части нетривиально), $H$ - максимальная почти слойно конечная подгруппа группы $G$, содержащая $C_{G}(i)$ (такая максимальная подгруппа найдется по лемме Цорна и ввиду почти слойной конечности локально конечных подгрупп в силу условий, накладываемых на группу $G$ (см. [20])).

Пусть $K$ - подгруппа из $H$, порожденная всеми инволюциями с бесконечными централизаторами в $H$. Слойно конечный радикал группы $H$ будем обозначать $R(H)$.

Дополнительно предположим, что группа $G$ обладает сильно вложенной подгруппой. При помощи свойств групп, обладающих сильно вложенной подгруппой (см., например, [19]) легко показать, что либо $H$ сильно вложена в $G$, либо обладает сильно вложенной подгруппой. Во втором случае для произвольной инволюции $k$ из $H$ справедливо равенство $k^{h}=i$ для некоторого элемента $h \in H$. Это означает, что в $H$ все инволюции имеют бесконечные централизаторы в $H$, и если $H \cap H^{g}$ содержит некоторую инволюцию, то по лемме $6 H=H^{g}$. Таким образом, подгруппа $H$ сильно вложена в группу $G$.

Обозначим через $\mathfrak{M}$ множество всех конечных подгрупп группы $G$ вида

$$
L_{g}=\operatorname{gr}\left(a, a^{g}\right)
$$

где элемент $a$ простого порядка $p$ выбираем из $H$, если $H$ - нечерниковская групта, и $a$ выбираем из нечерниковской почти слойно конечной подгруппы $B$, которая найдется в $G$ по теореме 3.1 из [22] и условиям, налагаемым на группу $G$, если группа $H$ черниковская ( $g \in G \backslash H$ в первом случае и $g \in G \backslash B$ во втором). Причем ввиду сопряженности инволюций в $G$ и сильной изолированности групп $B$ и $H$ в группе $B$ все инволюции почти регулярны. Группу $B$ можем считать максимальной почти слойно конечной подгруппой в групте $G$ ввиду леммы 3.

Ввиду бесконечности множества вариантов выбора порядка элемента $a$ и строения почти слойно конечной группы выберем его порядок таким достаточно большим, что он не делит индекс $|H: R(H)|$, где $R(H)$ - слойно конечный радикал группы $H$ в первом случае, и не делит индекс $|B: R(B)|$, где $R(B)$ - слойно конечный радикал группы $B$ во втором случае. Это можно сделать ввиду строения нечерниковской почти слойно конечной групшы. Во втором случае будем также предполагать, что $p \notin \pi(H)$ (это можно сделать ввиду черниковости $H$ ).

Лемма 13. Любая группа $V$ четного порядка из множества $\mathfrak{M}$ обладает сильно вложенной подгруппой и никакая сопряженная $c V$ подгруппа не лежит в $H$. 
Доказательство. Из-за сильной вложенности подгруппы $H$ все инволюции в группе $G$ сопряжены. Подберем элемент $b$ таким образом, чтобы группа $V^{b}$ содержала инволюцию $i$.

Пусть сначала $H$ - нечерниковская подгруппа. Предположим, что группа $V^{b}$ лежит в $H$. Тогда ввиду выбора числа $p$ элементы $a^{b}, a^{g b}$ содержатся в слойно конечном радикале группы $H$ и централизаторы $C_{H}\left(a^{g}\right)$ и $C_{H}\left(a^{g b}\right)$ бесконечны. Из леммы 6 и бесконечности $C_{H}(a)$ следует, что

$$
H=H^{b^{-1}}=H^{b^{-1} g^{-1}} .
$$

Но $H=N_{G}(H)$ в силу условий, накладываемых на группу $G$, и ввиду максимальности подгруппы $H$. Следовательно,

$$
b^{-1}, q b^{-1} g^{-1} \in H
$$

что влечет включение $g \in H$, но это противоречит выбору элемента $g$. Противоречие означает, что группа $V^{b}$ не лежит в $H$ и пересекается с ней нетривиально. В случае черниковской подгруппы $H$ группа $V^{b}$ не лежит в $H$ за счет выбора числа $p$. Таким образом, группа $V^{b}$ обладает сильно вложенной подгруппой, поэтому сильно вложенная подгруппа найдется и в группе $V$.

Заметим, что попутно мы доказали и вторую часть утверждения леммы. Лемма доказана.

Лемма 14. Можно считать, не нарушая общности рассуждений, что р выбрано настолько больиим, что группы $V \in \mathbb{M}$ не содержат инволючий.

Доказательство. Пусть $V$ - произвольная группа четного порядка из $\mathfrak{M}$. Она обладает сильно вложенной подгруппой по лемме 13 . По теореме Брауэра-Судзуки $[18,17]$ и по теореме Бендера (см. [18], стр. 142) $V / O_{2^{\prime}}(V)$ либо имеет единственную инволюцию, либо обладает нормальной подгруппой $F$ нечетного индекса, которая изоморфна одной из групп типа $P S L(2, K), S z(K), P S U(3, K)$, где $K$ - конечное поле характеристики 2.

Рассмотрим сначала вторую возможность. Так как силовские 2-подгруппы в $G$ сопряжены и являются черниковскими, порядок нижнего слоя силовской 2-подгруппы в группе $F$ ограничен (это элементарная абелева подгруппа). Тогда по свойствам групп $\operatorname{PSL}(2, K)$, $S z(K), P S U(3, K)$ их порядки ограничены некоторым числом, не зависящим от $p$.

Теперь, ввиду конечности множества порядков простых подгрупп $F$ в группе $V / O_{2^{\prime}}(V)$, выберем число $p$ настолько большим, что оно не делит порядков голоморфов этих подгрупп для всех подгрупп $V$ из $\mathfrak{M}$, то есть образы элементов $a, a^{g}$ в факторгруппе централизуют простую компоненту. Тогда они централизуют и силовскую 2-подгруппу $\bar{S}_{2}$ из $\bar{V}$. Ввиду того, что $O_{2^{\prime}}(V)$ - конечная $2^{\prime}$-подгруппа, найдутся элементы $b, c \in C_{G}\left(S_{2}\right)$ такие, что

$$
a \in b O_{2^{\prime}}(V), \quad a^{g} \in c O_{2^{\prime}}(V) .
$$

Ввиду сопряженности силовских 2-подгрупп в $G$ можно считать, что $S_{2}<H$. Тогда ввиду сильной вложенности $H$ элементы $b, c$ лежат в $H$. Так как нижний слой в группе $S_{2}$ - нециклическая элементарная абелева подгруппа, по теореме Брауэра [15]

$$
O_{2^{\prime}}(V)<H \text {. }
$$


Получаем включение $a, a^{g} \in H$, что противоречит лемме 13 .

Пусть имеет место первая возможность, то есть $V / O_{2^{\prime}}(V)$ обладает единственной инволюцией. В этом случае силовская 2-подгруппа в ней по теореме Бендера (см. [18], стр. 142) является либо циклической, либо обобщенной группой кватернионов, и по теореме Брауэра-Судзуки $[18,17]$ группа $V$ имеет вид

$$
V=O_{2^{\prime}}(V) C_{V}(k)
$$

где $k$ - некоторая инволюция из $V$.

Если $H$ - черниковская группа, то ввиду включения $C_{G}(k) \leqslant H$ и выбора числа $p \notin \pi(H)$ порождающие элементы группы $V$ принадлежат $O_{2^{\prime}}(V)$. Это вытекает из изоморфизма групп

$$
V / O_{2^{\prime}}(V), \quad C_{V}(k) / W
$$

где

$$
W=O_{2^{\prime}}(V) \cap C_{V}(k)
$$

Получили противоречие с тем, что группа $V$ содержит инволюции.

Пусть теперь $H$ - нечерниковская группа. Предположим, что $V$ неразрешима. Положим

$$
X=O_{2^{\prime}}(V) \cap C_{V}(k)
$$

По теореме об изоморфизме

$$
V / O_{2^{\prime}}(V) \cong C_{V}(k) / X
$$

Отсюда ввиду порождаемости $V$ элементами порядка $p$ и ее неразрешимости факторгруппа $V / O_{2^{\prime}}(V)$ также содержит элементы порядка $p$, а отсюда такие элементы содержит и факторгруппа $C_{V}(k) / X$, и значит, $C_{V}(k)$ обладает $p$-элементами.

Обозначим через $Z$ подгруппу, порожденную всеми $p$-элементами из $C_{V}(k)$. Ввиду сопряженности всех инволюций в $G$ инволюции $k, i$ сопряжены при помощи некоторого элемента $l$. Отсюда $C_{V}(k)<H^{l}$. Тогда $Z<H^{l}$. Еще немного ограничим возможность для выбора элемента $a$ : в случае нечерниковской группы $H$ будем предполагать, что $p$ не принадлежит конечному множеству $\pi\left(\left|H: C_{H}(K)\right|\right)$. Здесь через $K$ обозначено замыкание всех 2-элементов из $R(H)$ в $H$. Индекс $\left|H: C_{H}(K)\right|$ конечен ввиду черниковости силовской 2-подгруппы из $H$ и свойств слойно конечных групп. Заметим, что группа $C_{H}(K) \cap R(H)$ локально разрешима.

Теперь ввиду выбора числа $p$

$$
Z<C_{G}\left(K^{l}\right) \cap R\left(H^{l}\right) .
$$

Это означает, как мы показали выше, разрешимость $Z$. Чтобы получить разрешимость группы $V$ осталось заметить, что $C_{V}(k)=Z X$ или $V=O_{2^{\prime}}(V) Z$.

Покажем, что группа $V$ не может быть разрешимой. Действительно, в этом случае в фактор-группе $\bar{V}=V / O_{2^{\prime}}(V)$ максимальная нормальная 2-подгруппа централизуется образами порождающих элементов, следовательно, находится в ее центре и является циклической. В фактор-группе по ней очевидно уже нет нормальных 2-подгрупп, а ввиду разрешимости $V$ найдется нормальная элементарная абелева $q$-подгруппа $Q$ и $q \neq 2$. 
Учитывая строение группы автоморфизмов циклической 2-группы, видим, что полный прообраз в $\bar{V}$ группы $Q$ нормален в $\bar{V}$ и имеет вид $Q_{1} \times S$, где $Q_{1}-q$-подгруппа. Но тогда мы получили противоречие с построением подгруппы $O_{2^{\prime}}(V)$. Противоречие означает, что подгруппы $Q$ не найдется и $\bar{V}$ является 2-группой. Однако это вступает в противоречие с тем, что $V$ порождается $p$-элементами. Лемма доказана.

Лемма 15. Пусть $М$ - максимальная почти слойно конечная сильно вложенная подгруппа из $G, V$ - подгруппа, сопряженная с $M$ в $G, h$ - нетривиальный q-элемент из $D=M \cap V . E_{c л и} C_{V}(h)$ бесконечен, то и $C_{M}(h)$ бесконечен и наоборот.

Доказательство. Пусть $C_{M}(h)$ конечен и элементы

$$
g_{1}, g_{2}, \ldots, g_{n}, \ldots
$$

из $C_{V}(h)$ такие, что

$$
M_{1}, M_{2}, \ldots, M_{n}, \ldots
$$

- различные подгруппы вида $H_{n}=g_{n}^{-1} H g_{n}$, отличные от $M$. Так как $M$ - сильно вложенная подгруппа, элемент $g_{n}$ имеет представление $g_{n}=h_{n} t_{n}$, где $h_{n} \in M, t_{n}-$ инволюция, сопряженная с $i$ в $G$. Отсюда,

$$
M_{n}=t_{n} M t_{n}
$$

Обозначим через $D_{n}$ пересечение подгрупп $M$ и $M_{n}$. Пусть $P_{n}$ - силовская $q$-подгруппа из $D_{n}$, содержащая элемент $h$. Ввиду сильной вложенности $M$ группа $\operatorname{gr}\left(D_{n}, t_{n}\right)$ имеет представление

$$
D_{n} \lambda\left(t_{n}\right)=D_{n} \lambda\left(u_{n}\right),
$$

причем инволюцию $u_{n}$ подберем таким образом, чтобы она нормализовала $P_{n}$. Тогда $u_{n} \in N_{G}\left(Z\left(P_{n}\right)\right)$.

Среди подгрупп

$$
Z\left(P_{1}\right), Z\left(P_{2}\right), \ldots, Z\left(P_{n}\right), \ldots
$$

лишь конечное число различных ввиду конечности централизатора $C_{M}(h)$. Множество различных подтрупा

$$
P_{1}, P_{2}, \ldots, P_{n}, \ldots
$$

также не может быть бесконечным, иначе нашлась бы подгруппа $P_{m}$, у которой бесконечен $C_{M}\left(Z\left(P_{m}\right)\right)$, а это влечет включение

$$
u_{m} \in N_{G}\left(Z\left(P_{m}\right)\right) \leqslant M
$$

но тогда и $g_{m} \in M$ вопреки выбору $g_{m}$. Поэтому будем считать, не нарушая общности рассуждений, что

$$
P=P_{1}=P_{2}=\ldots=P_{n}=\ldots,
$$

и значит, в нормализаторе $N_{G}(P)$ содержится бесконечно много инволюций

$$
u_{1}, u_{2}, \ldots, u_{n}, \ldots
$$


Бесконечность централизатора $C_{V}(h)<V$ и конечность группы $P$, содержащей элемент $h$, влечет бесконечность пересечения $N_{G}(P) \cap V$. Отсюда, в силу условий, налагаемых на группу $G$, и леммы 4, группа $V$ содержит бесконечно много инволюций, что противоречит сильной вложенности группы $V$ и лемме 8. Противоречие означает, что $C_{M}(h)$ бесконечен. Лемма доказана.

Лемма 16. Если $H$ - нечерниковская сильно вложенная в $G$ подгруппа, то простое число р можно выбрать таким достаточно большим, что силовские $p$-подгруппы в $L_{g} \in \mathfrak{M}$ являются циклическими.

Доказательство. Пусть $H$ - нечерниковская группа, сильно вложенная в группу $G$. Рассмотрим подгруппы вида

$$
L_{g}=\operatorname{gr}\left(a, a^{g}\right) \in \mathfrak{M} .
$$

Так как $G$ является группой Шункова, подгруппы $L_{g}$ конечны. Обозначим через $P$ силовскую $p$-подгруппу из $L_{g}$, содержащую элемент $a$. Так как $P$, будучи конечной $p$-группой, обладает нетривиальным центром, выберем элемент $b$ простого порядка из $Z(P)$. Ввиду выбора элемента $a$ централизатор $C_{H}(b)$ бесконечен. Тогда по лемме $5 C_{G}(b) \leqslant H$. Следовательно, $P$ лежит в $H$.

Пусть $P$ не является циклической подгруппой. Обозначим элементарную абелеву подгруппу порядка $p^{2}$ из $P$, содержащую элемент $a$, через $R$. Рассмотрим подгруппу

$$
O_{p^{\prime}}\left(L_{g}\right) 入 R
$$

заметим, что $O_{p^{\prime}}\left(L_{g}\right) \neq 1$ по лемме 14 и теореме Файта-Томпсона. Согласно теореме Брауэра [15]

$$
O_{p^{\prime}}\left(L_{g}\right) \leqslant \operatorname{gr}\left(C_{G}(r) \mid r \in R^{\#}\right) .
$$

Элементы $r$ из $R^{\#}$ имеют бесконечные централизаторы в $H$ и в силу леммы 5 лежат в $H$ вместе с $O_{p^{\prime}}\left(L_{g}\right)$.

Проведя аналогичные рассуждения относительно подгруппы $H^{g}$ вместо $H$ и элемента $a^{g}$ вместо $a$, видим, что $O_{p^{\prime}}\left(L_{g}\right)<H^{g}$. Причем $p$ выбран настолько большим, что в централизаторах элементов порядка $p$ из $H$ нет элементов с конечными централизаторами в $H$, и это же справедливо для сопряженной подгруппы $H^{g}$.

Таким образом,

$$
O_{p^{\prime}}\left(L_{g}\right)<H \cap H^{g}
$$

и в $O_{p^{\prime}}\left(L_{g}\right)$ найдется $q$-элемент с бесконечным централизатором в $H$. Отсюда по лемме 15 централизатор элемента $h$ в $H^{g}$ также бесконечен и по лемме $6 H=H^{g}$. Получаем противоречие с выбором подгруппы $L_{g}$. Лемма доказана.

Лемма 17. Если $H$ - черниковская группа, то группа В почти абелева.

Доказательство. По свойствам слойно конечных групп (см., например, [7]) слойно конечный радикал $V$ группы $B$ обладает полной частью $A$, причем $A \leqslant Z(V)$. Как мы уже отмечали, в $B$ найдется почти регулярная инволюция $k$, которая ввиду свойств слойно конечных групп лежит в разности $B \backslash V$. В группе $V \lambda(k)$, очевидно, $A$ является нормальной подгруппой, причем $k$ действует регулярно на $A$ ввиду предложения 7 из [6]. 
Рассмотрим фактор-группу $\bar{V} \lambda(k A)$, где $\bar{V}=V / A$. Так как $\bar{V}$ не обладает бесконечными силовскими подгруппами, по свойствам слойно конечных групп с конечными (см., например, [7]) для каждого простого $p$ в $\bar{V}$ найдется $p^{\prime}$-подгруппа $\bar{V}_{p}$ конечного индекса, нормальная в $\bar{V} \lambda(i A)$. Пусть $\bar{C}$ - пересечение $\bar{V}_{p}$ по всем $p \in \pi\left(C_{\bar{V}}(k A)\right)$. Группа $\bar{C}$ имеет конечный индекс в $\bar{V}$ ввиду почти регулярности $k A$ в $\bar{V} \lambda(k A)$. По теореме Бернсайда (см., например, [13]) $\bar{C}$ как локально конечная группа, обладающая регулярным автоморфизмом порядка 2 , является абелевой группой. Ввиду локальной конечности $\bar{C} \lambda(k A)$ и свойств групп Фробениуса подгруппа $\bar{C}$ состоит из элементов, строго вещественных относительно $k A$.

Так как $A$ и $\bar{C}$ являются абелевыми 2-полными группами, по предложению 3.2 из [19] полный прообраз $C$ группы $\bar{C}$ в группе $V$ является абелевой группой. Поскольку она имеет конечный индекс в $B$, это доказывает утверждение леммы. Лемма доказана.

Замечание 1. Ввиду строения нечерниковской почти абелевой почти слойно конечной группы $B$ будем считать, что в случае черниковости группы $H$ число $p$ выбрано так, что оно не делит индекс $|B: L(B)|$, где $L(B)$ - нильпотентный радикал группы $B$ (этот индекс конечен, а множество $\pi(B)$ бесконечно ввиду строения бесконечной нечерниковской почти слойно конечной группы).

В дополнение к выбору числа $p$ согласно лемме 11 можем считать, что оно не принадлежит множеству

$$
\bigcup_{K} \pi\left(C_{B}(K)\right)
$$

где $K$ пробегает все элементарные абелевы подгруппы из $B$, имеющие в $B$ конечные централизаторы (это множество по лемме 11 конечно) в случае черниковской группы $H$; и в случае нечерниковской группы $H$ число $p \notin \pi\left(C_{H}(K)\right)$ для элементарных абелевых подгрупп $K$ из $H$ с конечными централизаторами в $H$.

Зафиксируем обозначение: в дальнейшем будем рассуждать об элементе $a$ из $B$ или из $H$ простого порядка, выбранном согласно замечанию.

Лемма 18. Если $H$ - черниковская сильно вложенная в $G$ подгруппа, то простое число р можно выбрать таким достаточно большим, что силовские р-подгруппы в

$$
L_{g}=\operatorname{gr}\left(a, a^{g}\right), \quad g \in G \backslash B,
$$

являются циклическими.

Доказательство. Так как $B$ - почти нильпотентная группа по лемме 17 , она имеет вид

$$
B=L(B) K
$$

где $L(B)$ - нильпотентный радикал группы $B, K$ - ее конечная подгруппа.

Рассмотрим подгруппы вида

$$
L_{g}=\operatorname{gr}\left(a, a^{g}\right), \quad g \in G \backslash B .
$$


Если лемма неверна, то, повторяя рассуждения из доказательства леммы 16 для подгруппы $B$ вместо $H$, получим включение $O_{p^{\prime}}\left(L_{g}\right) \leqslant B$, причем элементарная абелева подгруппа $R$, содержащая элемент $a$, лежит в $B$ ввиду включения $R \leqslant C_{G}(a)$. Таким образом,

$$
O_{p^{\prime}}\left(L_{g}\right) 入 R \leqslant B .
$$

Ввиду выбора $p$ подгруппа $R$ содержится в нильпотентном радикале $L(B)$ подгруппы $B$. Пусть $Q-$ силовская $q$-подгруппа из $O_{p^{\prime}}\left(L_{g}\right)$. В соответствии с леммой Фраттини [16] $Q$ выберем таким образом, чтобы $Q$ нормализовалось подгруппой $R$. Если $Q<L(B)$, то, очевидно, $Q \times R$. Если $q$ - делитель индекса $|B: L(B)|$, то, по определению нильпотентного радикала, $R$ нормализуется подгруппой $Q$. Таким образом, опять получаем, что $Q \times R$. Так как это рассуждение проходит для любого $q \in \pi\left(O_{p^{\prime}}\left(L_{g}\right)\right)$, заключаем, что

$$
O_{p^{\prime}}\left(L_{g}\right)<C_{G}(a) .
$$

Отсюда и ввиду выбора элемента $a$ все элементы из $O_{p^{\prime}}\left(L_{g}\right)$ имеют в $B$ бесконечные централизаторы, по лемме 5 целиком лежащие в $B$. Фиксируем произвольный элемент $c \neq 1$ из $O_{p^{\prime}}\left(L_{g}\right)$. Как показано выше,

$$
a \in C_{G}(c) \leqslant B
$$

Проведя аналогичные рассуждения относительно подгруппы $B^{g}$ вместо $B$ и элемента $a^{g}$ вместо $a$, видим, что

$$
a^{g} \in C_{G}(c), \quad c \in O_{p^{\prime}}\left(L_{g}\right) .
$$

Таким образом, $a^{g} \in B$. Ввиду выбора $p$

$$
\left|C_{G}\left(a^{g}\right) \cap B\right|=\infty .
$$

Очевидно, что $\left|C_{G}\left(a^{g}\right) \cap B^{g}\right|=\infty$. Значит, по лемме $6 B=B^{g}$ для элемента $g$ из $G \backslash B$. Получаем противоречие с выбором подгруппы $L_{g}$. Лемма доказана.

Доказательство теоремы 1. Пусть сначала $H$ - нечерниковская сильно вложенная подгруппа группы $G$. В силу леммы 16 считаем, не нарушая общности рассуждений, что найдется элемент $a \in H$ порядка $p$ такого, что силовская $p$-подгруппа в $L_{g}$ является циклической.

Предположим, что в $C_{L_{g}}(a)$ нашелся элемент $b$ простого порядка, который перестановочен с нетривиальным элементом $c$ из нильпотентного радикала $N_{g}$ группы $L_{g}=\operatorname{gr}\left(a, a^{g}\right)$.

По выбору порядка $p$ элемента $a$ элемент $b$ имеет бесконечный централизатор в $H$, значит, по лемме 5 он целиком содержится в $H$ вместе с элементом $c$. Отсюда следует, что пересеченне $D_{g}=N_{g} \cap H$ нетривиально, так как содержит элемент $c$. Рассмотрим максимальную нормальную элементарную абелеву $q$-подгруппу $A_{g}$ из $D_{g}$. Если $a$ действует регулярно на $A_{g}$, то ввиду строения группы регулярных автоморфизмов элементарной абелевой $q$-группы и конечности индекса слойно конечного радикала в почти слойно конечной группе за счет выбора $p$ добиваемся того, чтобы $q$ было настолько большим, что оно не делит индекс $|H: R(H)|$. Тогда $A_{g}<R(H)$. По свойствам слойно конечных групп и лемме 5

$$
C_{G}\left(A_{g}\right) \leqslant H
$$


Пусть теперь элемент $a$ перестановочен с нетривиальным элементом из $A_{g}$. Тогда либо он централизует всю $A_{g}$ и снова $C_{G}\left(A_{g}\right) \leqslant H$, либо $A_{g}$ расщепляется, так что $A_{g}=B_{g} \times C_{g}$, где $C_{g}<C_{G}(a)$, а на $B_{g}$ элемент $a$ действует регулярно. Тогда, как и выше, получаем ограничение на порядок $q: q$ не делит индекс $|H: R(H)|$.

Окончательно получаем независимо от действия $a$ на $A_{g}$ включение $C_{G}\left(A_{g}\right) \leqslant H$, что влечет по лемме 4 включение $N_{G}\left(A_{g}\right) \leqslant H$. Тогда $N_{G}\left(D_{g}\right) \leqslant H$.

Если $N_{g} \neq D_{g}$, то ввиду нормализаторного условия в нильпотентных группах нормализатор подгруппы $D_{g}$ в $N_{g}$ отличен от $D_{g}$ и по доказанному лежит в $H$. Получаем противоречие с построением $D_{g}$.

Если же $N_{g}=D_{g}$, то ввиду нормальности $N_{g}$ в $L_{g}$ и включения $N_{G}\left(D_{g}\right) \leqslant H$ получаем, что $L_{g}<H$, вопреки выбору группы $L_{g}$.

Таким образом, любой элемент простого порядка из $C_{L_{g}}(a)$ действует регулярно на $N_{g}$. Тогда по лемме 4.27 из [19] и лемме $16 L_{g}$ - группа Фробениуса с неинвариантным множителем (a). По основной теореме из [14] группа $G$ либо обладает нетривиальной нормальной локально конечной подгруппой вопреки лемме 2, либо имеет вид

$$
G=F 入 N_{G}((a))
$$

где $F \lambda(a)$ - группа Фробениуса. Если имеет место последняя ситуация, то противоречие с леммой 2 получается ввиду леммы 4.6 [22] и теоремы Шмидта.

Случай черниковской подгрупты $H$ рассматривается с учетом леммы 18 совершенно так же с заменой в рассуждениях подгруппы $H$ на подгруппу $B$. Теорема доказана.

Доказательство теоремы 2. Ввиду теоремы $1 H$ не является сильно вложенной подгруппой в группу $G$. Отсюда по лемме 6 сразу вытекает, что $H$ обладает почти регулярной инволюцией. Зафиксируем за этой инволюцией обозначение $j$. В локально конечных группах с черниковскими примарными подгруппами силовские примарные подгруппы сопряжены [21], поэтому инволюцию $j$ можно выбрать из $S$.

Напомним, что мы предполагаем группу $G$ периодической не почти слойно конечной группой Шункова и нормализатор любой ее нетривиальной конечной подгруппы почти слойно конечен. Предположим, что полная часть $\tilde{S}$ группы $S$ обладает больше, чем одной инволюцией, и кроме $i$ в $\tilde{S}$ нашлась инволюция $l$.

Напомним, что в $S$ найдется почти регулярная в $H$ инволюция $j$. Если $j l j=l$, то $(i) \times(l) \times(j)$ - элементарная абелева группа, существование которой противоречит лемме 9. Тогда $j l j=k \neq l$. Если $i k=l$, то поскольку мы находимся в полной части, можно выбрать элементы $l_{1}^{2}=l, k_{1}^{2}=k$ и одновременно $j l_{1} j=k_{1}$.

Тогда

$$
j l_{1} k_{1} j=k_{1} l_{1}=l_{1} k_{1}
$$

и порядок элемента $l_{1} k_{1}$ равен четырем. Продолжая рассуждения таким же способом, получаем противоречие с почти регулярностью инволюции $j$.

Остался случай, когда $i k \neq l$. Чтобы его исключить заметим, что

$$
j k l j=l k=k l
$$

а это как мы показали в начале доказательства невозможно при $k l \neq i$. 
Таким образом в полной части $\tilde{S}$ инволюция $i$ единственна. Отсюда следует, что $\tilde{S}$ является квазициклической 2-группой, которая содержится в слойно конечном радикале группы $H$, так как слойно конечный радикал имеет конечный индекс в $H$. Ввиду строения группы автоморфизмов квазициклической группы инволюция $j$ индуцирует в $\tilde{S}$ автоморфизм, переводящий каждый ее элемент в обратный. Если бы было справедливо неравенство

$$
C_{S}(\tilde{S}) \neq \tilde{S},
$$

то $\tilde{S}$ как полная абелева группа выделялась бы прямым множителем в некоторой большей абелевой подгруппе из $C_{S}(\tilde{S})$. Но тогда в $C_{S}(\tilde{S})$ нашлась бы инволюция $t$, не совпадающая с $i$, однако это невозможно, так как по свойствам слойно конечных групп $\tilde{S}$ нормальна в $H$, а по лемме 12 все инволюции с бесконечными централизаторами сопряжены в $H$. Следовательно,

$$
C_{S}(\tilde{S})=\tilde{S}
$$

а так как нетривиальный 2-автоморфизм группы $\tilde{S}$ может быть только второго порядка, To

$$
S=\tilde{S} \lambda(j)
$$

и $S$ - квазидиэдральная 2-группа. Теорема доказана.

\section{Список литературы}

1. Черников С. Н., К теории бесконечных р-групп. Докл. АН СССР. (1945) 50, 71-74.

2. Адян С. И., Проблема Бернсайда и тождества в группах. Наука, Москва, 1975.

3. Ольшанский А. Ю., Геометрия определяющих соотношений в группе. Наука, Москва, 1989.

4. Сенашов В. И., Группы с условием минимальности для не почти слойно конечных групп. Укр. матем. журн. (1991) 43, №7-8, 1002-1008.

5. Сенашов В. И., Почти слойная конечность периодической группы без инволюций. Укр. матем. журн. (1999) 51, №11, 1529-1533.

6. Шунков В. П., О периодических группах с почти регулярной инволюцией. Алгебра и логика (1972) 11, №4, 470-493.

7. Черников С. Н., Группы с заданными свойствами системы подгрупп. Наука, Москва, 1980.

8. Шунков В. П., О локально конечных группах конечного ранга. Алгебра и логика (1971) 10, №2, 199-225.

9. Шафиро А. А., Шунков В. П., Характеризация бесконечной черниковской группы, не являющейся конечным расширением квазициклической группы. Матем. сб. (1978) 107(149), №2(10), 289-303.

10. Каргаполов М. И., Локально конечные группы, обладающие нормальными системами с конечными факторами. Сиб. матем. журн. (1961) 2, №6, 853-873.

11. Созутов А. И., Шунков В. П., Об одном обобщении теоремы Фробениуса на бесконечные группы. Матем. сб. (1976) 100, №4, 495-508. 
12. Сучкова Н. Г., Шунков В. П., О группах с условием минимальности для абелевых подгрупп. Алгебра и логика (1986) 26, №4, 445-469.

13. Бусаркин В. М., Горчаков Ю. М., Конечные расщепляемые группы. Наука, Москва, 1968.

14. Созутов А. И., Шунков В. П., О бесконечных группах, насыщенных фробениусовыми подгруппами. Алгебра и логика (1977) 16, №6, 711-735.

15. Brauer R., Suzuki M., On finite groups with an abelian Sylow subgroups. Canad. J. Math. (1962) $14,436-450$.

16. Каргаполов М. И., Мерзляков Ю. И., Основы теории групп. Наука, Москва, 1982.

17. Hartley B., Finite groups of automrphisms of locally soluble groups. J. Algebra (1979) 57, №1, 242-257.

18. Kegel O. H., Wehrfritz B. A. F., Locally finite groups. North-Holland, Amsterdam, 1973.

19. Шунков В. П., $M_{p}$-группы. Наука, Москва, 1990.

20. Шунков В. П., Характеризация почти слойно конечных групп в классе локально конечных групп. В кн.: Теория групп. ВЦ СО РАН, Красноярск, 1996, с. 25-32.

21. Шунков В. П. О периодических группах с некоторыми условиями конечности. Докл. АН СССР (1970) 195, №6, 1290-1293.

22. Шунков В. П., О вложении примарных элементов в группе. Наука, Новосибирск, 1992.

Статья поступила 26.03.2002. 